\title{
Methods of calculation of energy losses of the refrigerator when opening its doors
}

\author{
S.P. Petrosov ${ }^{1}$, M.A. Lemeshko ${ }^{1 *}$, A.V. Kozhemyachenko ${ }^{1}$, S.E. Bashnjak ${ }^{2}$ \\ ${ }^{1}$ Institute of Service and Businesses (branch) DSTU "Don State Technical University", in Shakhty, Rostov region, 346500 \\ ${ }^{2}$ Don State Agrarian University, Persianovsky settlement, Rostov region, 346600
}

\begin{abstract}
The problem of energy supply and the problem of energy efficiency of energy consumers are global problems. In the modern world, scientists pay much attention to the tasks of creating new and improving traditional sources of electricity. The tasks of increasing the energy efficiency of electricity consumers remain relevant in the global economy. One of the relatively capacious energy consumers is the Park of refrigerators, household and commercial purposes, which are also being improved in order to increase their energy efficiency. Modernization and improvement of household and commercial refrigerators include the task of designing and using more efficient refrigerators. Energy consumption of compression refrigerators, along with other operational factors, depends on the processes of heat exchange and mass transfer of air from internal chambers to the ambient air. When the doors of the refrigerator chambers are opened, the cooled air in the chambers is replaced with the warmer air of the environment. This process causes the cost of operation of the refrigeration machine and characterizes its energy consumption. In order to minimize this power consumption, knowledge of the process of cool air outflow of their open refrigerator chambers is required. Currently, this process is poorly understood. The article presents an approach to the description of the process of motion of the cooled air from the refrigerator chamber, the main assumptions necessary for the construction of a mathematical model of this process are given, and a method for calculating the heat in the refrigerator chambers associated with the replacement of the cooled air with the ambient air inside the chambers is proposed. The method is based on the laws of gas dynamics, supplemented by some experimental studies. In particular, the well - known Navier-Stokes equation is used as the initial basis. This equation supplemented by the continuity equation of the air flow and other functional dependencies. As a result of mathematical transformations and arguments about the physics of the mass transfer process, finite equations are obtained, which allow describing both the performance of the cooled air flow and the amount of heat introduced into the chambers by external warm air. Mathematical dependences are developed for calculation of parameters of motion of a stream of the cooled air and heat inflows, thus the temperature of ambient air and temperature in chambers of the refrigerator are considered. The results of the research will be useful in the design of new refrigerating machines with reduced energy consumption.
\end{abstract}

\section{Introduction}

Household and commercial compression refrigerators are widely used in everyday life, in medicine, in trade, in public catering establishments, in hotel and restaurant business. The European standard EN 16001: 2009, adopted in most European countries, as well as the national standards of a number of states, regulates the requirements for reducing energy consumption of the used equipment, including the requirements for reducing energy consumption in household and commercial refrigerators. At the same time, it is important to solve the problems of reducing operating losses caused by opening the doors of refrigerating and freezing chambers.

The method of calculating heat inflows into the refrigerator chambers at opening doors is necessary for solving the problems of reducing energy consumption in this class of refrigerating machines [2]. 
Relatively warm environment air out of the refrigerator has lower density than the density of cooled air in the freezing or refrigerating compartments, causes the outflow of the cooled air from the refrigerating compartment. Essentially, it is the replacement of cooled air with warmer air [5].

Having determined the value and nature of changing cooled air outflow rates in the current moment when the door is opened, it is possible to determine the volume of replaced air and, consequently, energy costs necessary for restoring the temperature in the cooled compartment. For this purpose, the problem of calculating the air movement velocity when opening the refrigerator chambers [6] doors has been solved, and the technique for calculating heat inflow into the chambers has been developed [7,8]. Let us consider some general prerequisites and solutions of the pointed problem, considered by means of a physical model, partially described in the work [3].

\section{Development of a method}

Solving the tasks of regulating cooled air out flow from the refrigerator chambers is possible by means of using the laws of gas dynamics. To use these laws, some assumptions are adopted

- viscous gas motion is equal to the air motion is the main object of the present research.

-Gas is perfect, i.e., pressure $p$, density and absolute temperature

$\mathrm{T}$ satisfy the state of the equation of MendeleevClapeyron law

$$
p=\frac{\rho \cdot R_{0} \cdot T}{m},
$$

where: $R_{0}-$ is the universal gas constant;

$m-$ is molecular weight of the gas.

- The coefficient of specific heat "c" does not depend on the absolute temperature of the gas and is a physical constant of the gas.

- When the gas moves, only the viscosity of the first kind is taken into account (the resistance of the surrounding warm air to the cold air flow).

- The coefficient of thermal conductivity of the gas $« \lambda$ » is proportional to the coefficient of dynamic viscosity $\mu$, i.e, Prandtl criterion is satisfied

$$
\frac{\mu \cdot c}{\lambda}=\sigma=\text { const. }
$$

- The gas is "Newtonian" environment, subordinated to the well-known Newton generalized law about the linear connection between the stress tensor and the strain rate tensor.

- External forces do not affect the air flow inside of the refrigerator. For this model, one can consider the assumption

$$
F_{x}=0 ; F_{y}=0 ; F_{z}=-m g,
$$

where $g-$ is acceleration of gravity.

As there is the basic equation describing the motion of any viscous gas, the well-known Navier-Stokes equation describing the dynamics of a viscous gas per unit mass is used.

$$
\left\{\begin{array}{l}
\rho \frac{d u}{d t}=\rho F_{x}-\frac{\partial p}{\partial x}+\mu\left(\frac{\partial^{2} u}{\partial x^{2}}+\frac{\partial^{2} u}{\partial y^{2}}+\frac{\partial^{2} u}{\partial z^{2}}\right)+\mu \frac{\partial}{\partial x}(\operatorname{div} \bar{V})-\frac{2}{3} \frac{\partial}{\partial x}(\mu \cdot d i v \bar{V}) ; \\
\rho \frac{d v}{d t}=\rho F_{y}-\frac{\partial p}{\partial y}+\mu\left(\frac{\partial^{2} v}{\partial x^{2}}+\frac{\partial^{2} v}{\partial y^{2}}+\frac{\partial^{2} v}{\partial z^{2}}\right)+\mu \frac{\partial}{\partial y}(\operatorname{div} \bar{V})-\frac{2}{3} \frac{\partial}{\partial y}(\mu \cdot d i v \bar{V}) ; \\
\rho \frac{d w}{d t}=\rho F_{z}-\frac{\partial p}{\partial z}+\mu\left(\frac{\partial^{2} w}{\partial x^{2}}+\frac{\partial^{2} w}{\partial y^{2}}+\frac{\partial^{2} w}{\partial z^{2}}\right)+\mu \frac{\partial}{\partial z}(\operatorname{div} \bar{V})-\frac{2}{3} \frac{\partial}{\partial z}(\mu \cdot d i v \bar{V}) ;
\end{array}\right.
$$

where: $u, v, w-$ are the projection of the velocity vector $\bar{V}$ on the axis $\mathrm{OX}, \mathrm{Oy}, \mathrm{OZ}$;

$$
\bar{F}=\left(F_{x} ; F_{y} ; F_{z}\right)-\text { is the vector of external }
$$

volume forces acting on the gas at each point of space;

$$
\mu-\text { is the coefficient of dynamic viscosity; }
$$

$$
\operatorname{div} \bar{V}=\frac{\partial u}{\partial x}+\frac{\partial v}{\partial y}+\frac{\partial w}{\partial z}
$$

where:

$$
\begin{aligned}
& \frac{d u}{d t}=\frac{\partial u}{\partial t}+\frac{\partial u}{\partial x} u+\frac{\partial u}{\partial y} v+\frac{\partial u}{\partial z} w \\
& \frac{d v}{d t}=\frac{\partial v}{\partial t}+\frac{\partial v}{\partial x} u+\frac{\partial v}{\partial y} v+\frac{\partial v}{\partial z} w \\
& \frac{d w}{d t}=\frac{\partial w}{\partial t}+\frac{\partial w}{\partial x} u+\frac{\partial w}{\partial y} v+\frac{\partial w}{\partial z} w
\end{aligned}
$$

To the equations described above, the equation of the continuity of the air flow is added [4]:

$$
\frac{\partial \rho}{\partial t}+\frac{\partial(\rho u)}{\partial x}+\frac{\partial(\rho v)}{\partial y}+\frac{\partial(\rho w)}{\partial z}=0 .
$$

(6)

Under the above assumptions, the system of equations describing the air outflow from the refrigerating chambers contains seven unknown functions: $u, v, w, \rho, p, T, \mu$. In the above formulated problem six equations are proposed, as to the seventh equation which is essential for system closure, a diffusion equation or the heat balance equation can be proposed. 
Let us state the boundary conditions of the problem. Initial elementary values are to be taken as an example of real calculations.

1) Equality of zero velocity is situated on a fixed rigid boundary inside of the refrigerator cabinet

2) The initial cold air flow velocity from the cabinet is zero at each point of the rectangular section at the exit of the cabinet.

3) The set air temperature inside of the cabinet at the initial moment of cold air outflow from the cabinet is equal to $T_{\text {хол }}=5^{0} \mathrm{C}$.

4) At the initial time, the temperature outside of the refrigerating cabinet is constant and equal to $T_{\text {комн }}=25^{\circ} \mathrm{C}$.

5) The air velocities outside the refrigeration unit at the initial moment of opening the door at each point of space are zero..

At the first stage, to simplify the task of cold air outflow from the refrigerating cabinet, it is additionally supposed that the process is isothermal, i.e, the temperature of freely air outflow is constant at each point of the air outflow. In fact, such condition means that the diffusion process of mixing cold (freely outflow) air and warm (room) is excluded. It is also assumed that the cold air outflow is the incompressible (non-expanding) environment.

Under such assumptions, the following equalities are evident

$$
T=\text { const }_{1}, p=\text { const }_{2}, \mu=\text { const }_{3} .
$$

Since the airflow is assumed to be incompressible, so

$$
\operatorname{div} \bar{V}=\frac{\partial u}{\partial x}+\frac{\partial v}{\partial y}+\frac{\partial w}{\partial z} \equiv 0
$$

Under the assumptions made, the continuity equation is satisfied.

By the condition (8) the last two terms in each equation of the system (4) are equal to zero by means of using formulas (1), (4) and assumptions(6), (7), system (5) can be transformed to a simpler form:

$$
\left\{\begin{array}{l}
\rho\left(\frac{\partial u}{\partial t}+\frac{\partial u}{\partial x} u+\frac{\partial u}{\partial y} v+\frac{\partial u}{\partial z} w\right)=\mu\left(\frac{\partial^{2} u}{\partial x^{2}}+\frac{\partial^{2} u}{\partial y^{2}}+\frac{\partial^{2} u}{\partial z^{2}}\right) \\
\rho\left(\frac{\partial v}{\partial t}+\frac{\partial v}{\partial x} u+\frac{\partial v}{\partial y} v+\frac{\partial v}{\partial z} w\right)=\mu\left(\frac{\partial^{2} v}{\partial x^{2}}+\frac{\partial^{2} v}{\partial y^{2}}+\frac{\partial^{2} v}{\partial z^{2}}\right) \\
\rho\left(\frac{\partial w}{\partial t}+\frac{\partial w}{\partial x} u+\frac{\partial w}{\partial y} v+\frac{\partial w}{\partial z} w\right)=-\rho m g+\mu\left(\frac{\partial^{2} w}{\partial x^{2}}+\frac{\partial^{2} w}{\partial y^{2}}+\frac{\partial^{2} w}{\partial z^{2}}\right)
\end{array}\right.
$$

After the above assumptions, the system (9) is a system of three equations relative to three unknown functions $u, v, w$, i.e., the system (9) is closed.

It is also assumed that the cold air outflow from the cabinet occurs without changes in the horizontal transverse direction i.e. that the ordinates of the trajectories of the air flow motion are constant and the change in the geometry of the flow occurs only in the plane XOZ (Figure 1).

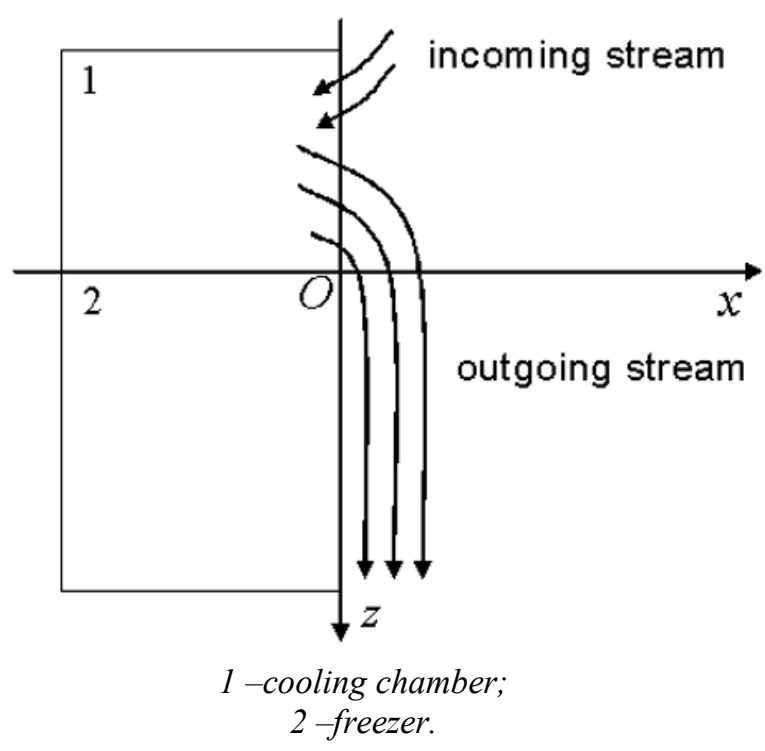

Fig. 1. The air flows motion with the open door of the fridge in the plane $\mathrm{XOZ}$

The assumption of the geometry invariability of the flow in the direction of the axis OY implies that the values of the function vanish $v=v(x, y, z) \equiv 0$ and functions are $u=u(x, z) ; w=w(x, z)$, i.e. they do not depend on the variable « $y »$. Thus, the real three-dimensional flow is replaced by the twodimensional model

Under these conditions, the system (9) is simplified

$$
\left\{\begin{array}{l}
\rho\left(\frac{\partial u}{\partial t}+\frac{\partial u}{\partial x} u+\frac{\partial u}{\partial z} w\right)=\mu\left(\frac{\partial^{2} u}{\partial x^{2}}+\frac{\partial^{2} u}{\partial z^{2}}\right) \\
\rho\left(\frac{\partial w}{\partial t}+\frac{\partial w}{\partial x} u+\frac{\partial w}{\partial z} w\right)=\rho m g+\mu\left(\frac{\partial^{2} w}{\partial x^{2}}+\frac{\partial^{2} w}{\partial z^{2}}\right) .
\end{array}\right.
$$

in addition the process of the air outflow is supposed to be stationary in time, then

$$
\frac{\partial u}{\partial t} \equiv 0, \frac{\partial w}{\partial t} \equiv 0
$$

and the system (10) becomes simpler in the form 


$$
\left\{\begin{array}{l}
\left(\frac{\partial u}{\partial x} u+\frac{\partial u}{\partial z} w\right)=\frac{\mu}{\rho}\left(\frac{\partial^{2} u}{\partial x^{2}}+\frac{\partial^{2} u}{\partial z^{2}}\right) \\
\left(\frac{\partial w}{\partial x} u+\frac{\partial w}{\partial z} w\right)=m g+\frac{\mu}{\rho}\left(\frac{\partial^{2} w}{\partial x^{2}}+\frac{\partial^{2} w}{\partial z^{2}}\right) .
\end{array}\right.
$$

Since the system (12) contains two equations and two unknown functions, it is closed.

The system (12) can be classified as a system of two nonlinear partial differential equations of the second order. At the present time methods of solving such problems are unknown in technical literature. In this paper, the system (12) is proposed to be solved by means of approximate analytical methods.

The expressions, in both equations of the system (12) determine the forces of aerodynamic resistance of the air environment of the room, which delays the cold air flow, flowing out of the refrigerating chamber.

The system of equations (12) will be transformed proceeding from the fact that in technical aerodynamics the resistance force of the air environment is approximately determined by the formulas (in the socalled zone of quadratic resistance) [9].

$$
\begin{gathered}
\frac{\mu}{\rho}\left(\frac{\partial^{2} u}{\partial x^{2}}+\frac{\partial^{2} u}{\partial z^{2}}\right), \frac{\mu}{\rho}\left(\frac{\partial^{2} w}{\partial x^{2}}+\frac{\partial^{2} w}{\partial z^{2}}\right) \\
\frac{\mu}{\rho}\left(\frac{\partial^{2} u}{\partial x^{2}}+\frac{\partial^{2} u}{\partial z^{2}}\right) \approx-\frac{\mu k}{\rho} u^{2} ; \\
\frac{\mu}{\rho}\left(\frac{\partial^{2} w}{\partial x^{2}}+\frac{\partial^{2} w}{\partial z^{2}}\right) \approx-\frac{\mu k}{\rho} w^{2},
\end{gathered}
$$

here $k-$ is the coefficient determined experimentally.

Since the coefficient of dynamic viscosity $\mu$ has units of measure $\mathrm{kg} \frac{\kappa 2}{\mathcal{M} \cdot \boldsymbol{C}}$, then from equations (13)we get that, $k$ is measured in $-\frac{C}{M^{3}}$.

The minus in the right-hand side of equalities (13) indicates that the second partial derivatives of the projections of the velocity vector are negative because of the inhibitory effect of the viscous environment.

Using formulas (13) the system (12) is transformed in the form

$$
\left\{\begin{array}{l}
\left(\frac{\partial u}{\partial x} u+\frac{\partial u}{\partial z} w\right)=-\frac{\mu k}{\rho} u^{2} \\
\left(\frac{\partial w}{\partial x} u+\frac{\partial w}{\partial z} w\right)=m g-\frac{\mu k}{\rho} w^{2} .
\end{array}\right.
$$

The system (14) is basic for determining air flow velocities when it flows freely out of the refrigerating chamber in a stationary mode.

Let us consider that the free air flow from the refrigerator cabinet depends on the time of outflow. First, let us investigate the behavior of the free air flow inside of the refrigerating chamber. Since the bottom of the refrigerating chamber is horizontal, the airflow inside of the chamber is supposed to be onedimensional i.e. the only horizontal component of the velocity vector will be affected $u=u(x ; t)$, and the values of the vertical component will be neglected, which means

$$
w=w(x ; t)=0 .
$$

Gravity forces acting on the horizontal projection of the velocity vector for the air flow inside of the refrigerating chamber will not be taken into account.

In the basic system of equations of the air flow motion under the condition (15) the second equation is identically satisfied, taking into account (15) the system of equations (10), describing the non stationary motion of cooled air, transformed into one equation relative to the unknown function $u=u(x ; t)$

$$
\frac{\partial u}{\partial t}+\frac{\partial u}{\partial x} u=\frac{\mu}{\rho} \frac{\partial^{2} u}{\partial x^{2}} \text {. }
$$

If we apply the same transformation to the equation (16) as for the system (12), the equation (16) will be transformed proceeding from the fact that in technical aerodynamics the resistance force of the air environment is approximately determined by the formula (in the zone of quadratic resistance)

$$
\frac{\mu}{\rho} \frac{\partial^{2} u}{\partial x^{2}} \approx-\frac{k \mu}{\rho} u^{2} \text {. }
$$

Paying attention to the formula (17) the equation (16) after transferring all expressions of the equation to the left-hand side, it takes the form

$$
\frac{\partial u}{\partial t}+\frac{\partial u}{\partial x} u+\frac{k \mu}{\rho} u^{2}=0 .
$$

Approximate solutions of the equation (18) will be found in the form

$$
u(x ; t)=b_{0}(t)+b_{1}(t) e^{-x},
$$

where $b_{0}(t), b_{1}(t)-$ are new unknown functions from a variable $« t »$.

To find the solution of equation (18), let substitute it into the function $u=u(x ; t)$ in the form (19), after simplifying we obtain the following: 


$$
\begin{aligned}
& \frac{d b_{0}(t)}{d t}+\frac{\mu k}{\rho} b_{0}^{2}(t)+\left(\frac{d b_{1}(t)}{d t}+\left(2 \frac{\mu k}{\rho}-1\right) b_{0}(t) b_{1}(t)\right) e^{-x}+ \\
& +\left(\frac{\mu k}{\rho}-1\right) b_{1}^{2}(t) e^{-2 x}=0 .
\end{aligned}
$$

Equating the "free term" of equation to zero (20 73) paying attention to the degrees of the function, $e^{-x}$, we obtain

$$
\frac{d b_{0}(t)}{d t}+\frac{\mu k}{\rho} b_{0}^{2}(t)=0 .
$$

The equation (21) is an ordinary differential equation with separating variables, the general solution has the form

$$
b_{0}(t)=\frac{1}{\frac{\mu k}{\rho} t+C_{1}},
$$

where $C_{1}-$ is an arbitrary constant.

to find the function $b_{1}(t)$ the expression for the function $e^{-x}$ is equated to zero in the equation (20-73)

$$
\frac{d b_{1}(t)}{d t}+\left(2 \frac{\mu k}{\rho}-1\right) b_{0}(t) b_{1}(t)=0
$$

In the equation (23), instead of the function $b_{0}(t)$, we substitute the function (22), which is a solution of equation (21)

$$
\frac{d b_{1}(t)}{d t}+\left(2 \frac{\mu k}{\rho}-1\right) \frac{b_{1}(t)}{\frac{\mu k}{\rho} t+C_{1}}=0 .
$$

The equation (24) is also an equation with separable variables with general solution

$$
b_{1}(t)=C_{2}\left(\frac{\mu k}{\rho} t+C_{1}\right)^{-2+\frac{\rho}{\mu k}}
$$

$C_{2}-$ is arbitrary constant

Thus, an approximate solution of equation (18) is obtained in the form (19) up to terms of order $e^{-2 x}$, where the functions $b_{0}(t)$ and $b_{1}(t)$ are determined by formulas (22), (25)

The values of the constants $\mathrm{C} 1$ and $\mathrm{C} 2$ can be found according to the initial conditions by the well known method. As a result of the calculations, it is obtained:

$$
\left\{\begin{array}{c}
C_{1}=\frac{\mu k-\rho}{U_{0}(2 \mu k-\rho)} ; \\
C_{2}=-\frac{\mu k \cdot U_{0}\left(\frac{\mu k-\rho}{U_{0}(2 \mu k-\rho)}\right)^{2-\frac{\rho}{\mu k}}}{\mu k-\rho} .
\end{array}\right.
$$

Thus, in the form (19) an approximate solution of the task is obtained for determining the airflow rates inside of the refrigerating chambers where the functions $b_{0}(t), b_{1}(t)$, are determined by formulas (22), (25), but arbitrary constants $C_{1}, C_{2}-$ are according to the formulas (26).

It was found experimentally that the quantity is $\mu k-\rho>0$, therefore inequalities are

$$
C_{1}>0, C_{2}<0 \text {. }
$$

We define the sign of the function as $u(x ; t)$. For this we do substitutes in the expression (19) functions $b_{0}(t), b_{1}(t)$ from (22), (25) and the values of the constants $C_{1}, C_{2}$ in accordance to (26), so after simplifications, we get

$$
u(x ; t)=\frac{1}{\frac{\mu k}{\rho} t+\frac{\mu k-\rho}{U_{0}(2 \mu k-\rho)}}-\frac{\left(\frac{\mu k-\rho}{U_{0}(2 \mu k-\rho)}\right)^{2-\frac{\rho}{\mu k}}}{\left(U_{0}\right)^{-1}\left(1-\frac{\rho}{\mu k}\right) e^{x}}\left(\frac{\mu k}{\rho} t+\frac{\mu k-\rho}{U_{0}(2 \mu k-\rho)}\right)^{-2+\frac{\rho}{\mu k}} .
$$

Let us transform the function (27) to a convenient form for calculations

$$
u(x, t)=\frac{U_{0}\left(2-\frac{\rho}{\mu k}\right)}{\left(\frac{\mu k U_{0}(2 \mu k-\rho)}{\rho(\mu k-\rho)} t+1\right)\left(1-\frac{\rho}{\mu k}\right)}-\frac{U_{0} e^{-x}}{\left(1-\frac{\rho}{\mu k}\right)}\left(\frac{\mu k U_{0}(2 \mu k-\rho)}{\rho(\mu k-\rho)} t+1\right)^{-2+\frac{\rho}{\mu k}} .
$$

In the expression (28) we change the variable

$$
\frac{\mu k U_{0}(2 \mu k-\rho)}{\rho(\mu k-\rho)} t=\xi \text {. }
$$

After the substitution (29), the function $u(x ; t)$ will take the form

$$
u(x ; \xi)=\frac{U_{0}}{\left(1-\frac{\rho}{\mu k}\right)}\left(\left(2-\frac{\rho}{\mu k}\right)(\xi+1)^{-1}-(\xi+1)^{-2+\frac{\rho}{\mu k}} e^{-x}\right)
$$

We represent the function (30) in the form: 
$u(x ; \xi)=\frac{U_{0}}{\left(1-\frac{\rho}{\mu k}\right)}\left(\left(1-\frac{\rho}{\mu k}\right)(\xi+1)^{-1}+(\xi+1)^{-1}\left(1-(\xi+1)^{-2+\frac{\rho}{\mu k} e^{-x}}\right)\right)$.

As $\mu k-\rho>0, \quad$ so $1-\frac{\rho}{\mu k}>0$. From the substitution (28) it is easy to see that $\xi>0$, then expression $(\xi+1)^{-2+\frac{\rho}{\mu k}} e^{-x}<1$ and function (31) are positive for all « $x »$ and $\xi>0$, the function $u(x ; t)$ is positive for all $« x »$ and $t>0$.

Let us clarify the sign of the derivative of the function $u(x ; t)$. We differentiate the function $u(x ; \xi)$ relative to the variable « $\xi »$

$$
\frac{\partial u(x ; \xi)}{\partial \xi}=\frac{U_{0}}{\left(1-\frac{\rho}{\mu k}\right)}\left(-\left(2-\frac{\rho}{\mu k}\right)(\xi+1)^{-2}-\left(-2+\frac{\rho}{\mu k}\right)(\xi+1)^{-3+\frac{\rho}{\mu k}} e^{-x}\right),
$$

or

$$
\frac{\partial u(x ; \xi)}{\partial \xi}=-\frac{U_{0}\left(2-\frac{\rho}{\mu k}\right)}{\left(1-\frac{\rho}{\mu k}\right)(\xi+1)^{2}}\left(1-(\xi+1)^{-1+\frac{\rho}{\mu k}} e^{-x}\right)
$$

From the received representation (32), it is easy to see that each factor on the right-hand side of the equation is positive, i.e.. $\frac{\partial u(x ; \xi)}{\partial \xi}<0$ for all $x$ » и $\xi>0$. It follows from the linearity of the substitution (28) that expressed as $\frac{\partial u(x ; t)}{\partial t}<0$ for all «x» are $t>0$.

Thus the function $u(x ; t)$ is positive for all $x$ » и $t>0$, as it monotonically decreases in time, i.e, the velocity of air outflow decreases with time. As a result of experimental studies it was obtained that the height of the air outflow from the chambers at the exit is changed with time by the law

$$
h(0 ; t)=\frac{H_{1}}{3} e^{-\alpha t} .
$$

The value of the parameter $\alpha$ was determined experimentally, it turned out to be equal to $\alpha=0,21$. value $h(0 ; t)=\frac{H_{1}}{3} e^{-\alpha t}$.
Let us determine the flow rate of chilled air freely flowing out of the chamber. According to [8], the flow rate of the investigated air flow at the exit of the chamber is determined by the formula

$$
\Pi=h(0 ; t) \cdot u(0 ; t) \cdot b,
$$

where $b-$ is refrigerating chamber width.

In the experiment, the value is $b=0,5 \mathrm{M}$.

Using the formula (28) we obtain

$u(0 ; t)=\frac{U_{0}}{\left(1-\frac{\rho}{\mu k}\right)}\left(\frac{\left(2-\frac{\rho}{\mu k}\right)}{(\beta t+1)}-(\beta t+1)^{-2+\frac{\rho}{\mu k}}\right)$.

where $\beta=\frac{\mu k U_{0}(2 \mu k-\rho)}{\rho(\mu k-\rho)}$.

Thus, the flow consumption (rate) is determined by formula (34), where the functions $h(0 ; t)$ and $u(0 ; t)$ are determined by formulas (33), (35). Heat loss in the free air outflow from the refrigerating chamber is determined by the formula [1]:

$$
Q=c \cdot \Pi \cdot \rho \cdot\left(T_{x}-T_{\Pi}\right)
$$

where $C-$ is specific air heat

Using the formulas (33) we get

$$
Q=c \cdot \rho \cdot h(0 ; t) \cdot u(0 ; t) \cdot b \cdot\left(T_{x}-T_{\Pi}\right)
$$

Thus, according to the formula (37), the heat loss for the cold chamber is determined.

\section{Conclusions and recommendations}

1. The basis of the method to determine heat inflows into the chambers of the refrigerator, after opening the doors, is the Navier-Stokes equation, which is added by the equation of motion continuity.

2. To solve the problem, well-founded assumptions are made, to obtain a closed system of equations.

3. In order to carry out practical calculations, a threedimensional model describing the air motion is transferred into an adequate two-dimensional model.

4. The obtained mathematical expressions allow us to calculate the outflow of cooled air from the refrigerator compartment and the amount of heat (heat loss) with free air outflow from the refrigerating compartment when the refrigerator doors are opened. 


\section{References}

1. A. P. Baskakov, B. V. Berg, O. K. Vitt - M.: Energoatomizdat, (1991)

2. R.O. Dating. Refrigerants: Service Pointers. - Refrigeration Service and Contracting. 39. (1971)

3. Y.G. Fomin, et al. Mathematical model of heat leakage in Camera of Refrigerator 1 (361) News of higher educational institutions. Technology of textile industry. S. Ivanovo, 158-161. (2016)

4. L.G. Loitsyansky. Mechanics of liquids and gases (Nauka. Moscow, 1978)

5. M.A. Lemeshko, Z.V. Gornostaeva, E.V. Duvanskaya, I.N. Danileko. Determination of the projections of the velocity Vector of the cooled Air from the Refrigerating Compartment with the door open. ARPN Journal of Engineering and Applied Sciences. 10(2), 954-958. (2015)

6. M.A. Lemeshko, E.V. Duvanskaya, S.P. Petrosov, V.N. Kohanenko. Mathematical Model of Refrig- erated Air from the Fridge Speed Calculation. World Applied Sciences Journal, 30(9):1145-1151 (2004)

7. M.A. Lemeshko, V.V. Laletin M.F. Mizik. Determination of the speeds of the movement of cooled air when opening the doors of the cabinet of a domestic refrigeration appliance [Electronic resource] // "Engineering Bulletin", № 4. (2011). available from: http://www.ivdon.ru/magazine/archive/n4y2011

8. M.A. Lemeshko, M.F. Mizik, Mathematical model of free flow of cooled air from the chamber of a domestic refrigeration device. / Izvestiya of higher educational institutions. North-Caucasian region. Series: Engineering. 4 (173) 16-18. (2013)

9. S.A. Chaplygin, Izbrannye trudy. Mekhanika zhidkosti i gaza, matematika, obshchaya mekhanika (Selected Works. Mechanics of Fluids and Gas, Mathematics, General Mechanics), (Nauka: Moscow, 1976) 